\title{
Étude de l'emprisonnement des raies de résonance dans les mélanges de gaz rares
}

\author{
J.P. Gardou, F. Marchal, N. Sewraj, S. Tusseau, P. Millet et Y. Salamero \\ Centre de Physique des Plasmas et de leurs Applications de Toulouse, ESA 5002, \\ Université Paul Sabatier, 118 route de Narbonne, 31062 Toulouse cedex, France
}

\begin{abstract}
When one component of a binary mixture is selectively excited on a resonant state, the interaction between excited and ground state atoms broadens the line profile. Here, the broadening coefficient of the $123,58 \mathrm{~nm}$ krypton resonant line, due to $\mathrm{Kr}-\mathrm{Ar}$ collisions, is determined experimentally from measurements of the imprisonment time of the resonant state.
\end{abstract}

\section{INTRODUCTION}

La mesure des durées de vie apparentes des états résonnants les plus bas des gaz rares permet la détermination des coefficients d'élargissement de la transition résonnante dus à l'introduction d'un gaz rare plus léger. L'étude porte sur l'élargissement de la transition résonnante $\operatorname{Kr}\left({ }^{3} \mathrm{P}_{1} \rightarrow{ }^{1} \mathrm{~S}_{0}\right)$ à $123,58 \mathrm{~nm}$ en présence d'argon.

L'excitation initiale est réalisée de façon sélective sur l'état atomique $\mathrm{Kr}\left({ }^{3} \mathrm{P}_{1}\right)$ par absorption multiphotonique à trois photons à partir de l'état fondamental $\operatorname{Kr}\left({ }^{1} \mathbf{S}_{0}\right)$. L'enregistrement du déclin de la luminescence est effectué derrière un filtre interférentiel centré à $128 \mathrm{~nm}(\mathrm{FWHM}=10 \mathrm{~nm})$ au moyen d'un photomultiplicateur 'extremely solar blind' travaillant en régime de détection par photoélectron unique [1].

\section{SUPPORT THEORIQUE}

Dans le cadre d'un élargissement de pression, la mi-largeur à mi-hauteur du profil d'absorption a l'expression suivante [2] ( $n_{0}$ est la densité d'atomes sur l'état fondamental, $v_{0}$ est la fréquence centrale de la raie, $g_{1}$ et $g_{2}$ sont respectivement les poids statistiques du niveau fondamental et de l'état résonnant, $f$ est la force d'oscillateur, les autres paramètres sont les constantes habituelles) :

$$
\gamma=\left(\frac{g_{1}}{g_{2}}\right)^{1 / 2} \frac{e^{2} \mathbf{f}}{11,7 \cdot \pi^{2} \cdot \varepsilon_{0} \cdot m_{e} \cdot v_{0}} n_{0}=\xi_{K r} \cdot n_{0}
$$

$\xi_{\mathrm{Kr}}$ est le coefficient d'élargissement du profil d'émission de la raie dû aux collisions entre atomes de krypton. Dans le cas d'un mélange de krypton et d'argon, les collisions entre atomes d'argon et de krypton contribuent à un élargissement supplémentaire de la raie de résonance. On introduit donc le coefficient d'élargissement $\xi_{\mathrm{Ar}}$ dû à la présence d'argon. La mi-largeur à mi-hauteur du profil lorentzien résultant est proportionnelle aux densités des deux constituants du mélange :

$$
\gamma=\xi_{\mathrm{Kr}} \mathrm{n}_{\mathrm{OXe}}+\xi_{\mathrm{Ar}} \mathrm{n}_{\mathrm{OAr}}
$$


Dans nos conditions, on peut considérer seulement l'élargissement de pression.

La durée de vie apparente de l'état résonnant $\operatorname{Kr}\left({ }^{3} \mathrm{P}_{1}\right)$ est fonction de la concentration $\mathrm{C}$ du krypton dans le mélange et peut se mettre sous la forme suivante [3] :

$$
\tau_{\mathrm{a}}=\mathrm{A}\left(\frac{\mathrm{C}}{\mathrm{C} \xi_{\mathrm{Kr}}+(1-\mathrm{C}) \xi_{\mathrm{Ar}}}\right)^{1 / 2}
$$

Le coefficient $A$ tient compte de la transition impliquée et du facteur géométrique de l'enceinte contenant le mélange gazeux. Dans nos conditions d'expérience ce coefficient n'est pas aisé à déterminer à cause de la géométrie complexe de la cellule. Toutefois il n'intervient pas dans le rapport des durées de vie apparentes pour deux concentrations distinctes de krypton. Dans le cas où $\tau_{\mathrm{al}}$ représente la durée de vie apparente mesurée pour une pression donnée de krypton pur, ce rapport permet d'obtenir le coefficient d'élargissement dû à l'argon :

$$
\xi_{\mathrm{Ar}}=\frac{\mathrm{C}}{1-\mathrm{C}} \xi_{\mathrm{Kr}}\left(\frac{\tau_{\mathrm{a} 1}{ }^{2}}{\tau_{\mathrm{a} 2}{ }^{2}}-1\right)
$$

$\tau_{\mathrm{a} 2}$ est la durée de vie apparente mesurée pour une concentration $\mathrm{C}$ de krypton.

\section{RESULTATS EXPERIMENTAUX}
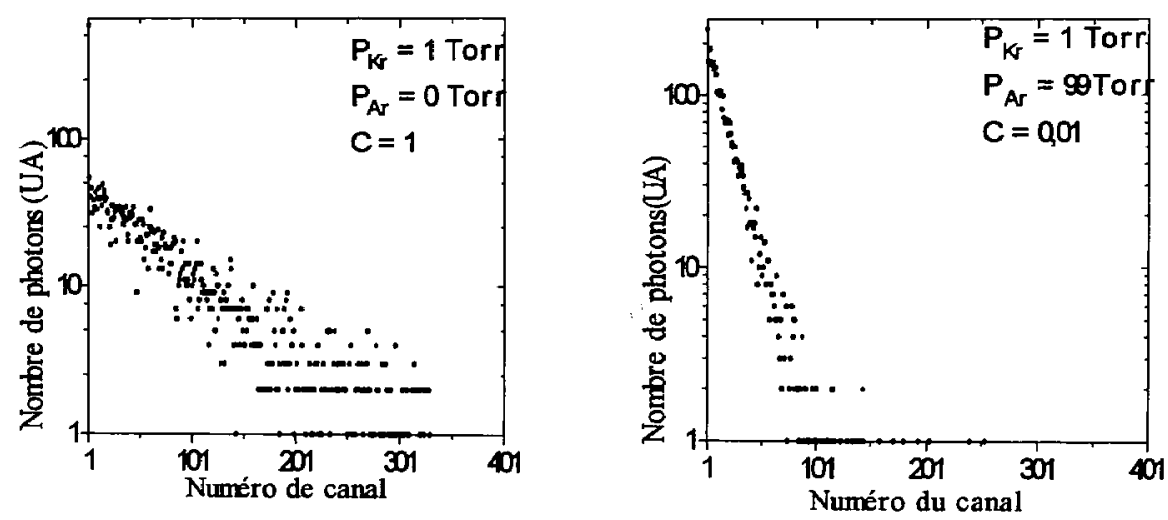

Evolution du déclin de la luminescence pour $\mathrm{C}=1$ et $\mathrm{C}=0,01$

L'exploitation du déclin de la luminescence en un terme exponentiel permet d'obtenir la valeur de la durée de vie apparente qui vaut respectivement $3,95 \mu$ s et $0,88 \mu$ s pour $C=1$ et $\mathrm{C}=0,01$. On en déduit la valeur du coefficient d'élargissement dû à l'argon :

$$
\xi_{\mathrm{Ar}}=1,9310^{-16} \mathrm{~m}^{3} \mathrm{~s}^{-1} \quad\left(\operatorname{avec} \xi_{\mathrm{Kr}}=9,84510^{-16} \mathrm{~m}^{3} \mathrm{~s}^{-1}\right)
$$

\section{Références}

[1] Berejny P., Millet P., Saissac M. and Salamero Y., J. Phys. B, 26, (1993) 3339-3353

[2] W. Lochte-Holtgreven, Plasma Diagnostics (North Holland Publishing Company, Netherlands, 1968)

[3] Igarashi K., Mikoshiba S., Watanabe Y., Suzuki M. and Muruyama S., J. Phys. DApplied Phys, 28, (1995) 1377-1383 